\title{
Erratum to: On the Extremal Zagreb Indices of Graphs with Cut Edges
}

\section{Yanqin Feng • Xia Hu $\cdot$ Shuchao Li}

Published online: 9 February 2010

(C) Springer Science+Business Media B.V. 2010

\section{Erratum to: Acta Appl Math}

DOI 10.1007/s10440-009-9467-8

We correct Theorem 5.2, which relates the sharp upper bound for the second Zagreb index of graphs with $k$ cut edges, as the following:

Theorem 5.2 Let $G \in \mathcal{G}_{n}^{k}$, then

$$
4 n+4 \leq M_{2}(G) \leq \frac{1}{2}(n-k-1)^{3}(n-k-2)+(n-1)\left[(n-k-1)^{2}+k\right],
$$

the left equality holds if and only if $G \cong P_{n}^{k}$ and the right equality holds if and only if $G \cong K_{n}^{k}$.

The online version of the original article can be found under doi:10.1007/s10440-009-9467-8.

Y. Feng $\cdot$ X. Hu

School of Mathematics and Statistics, Wuhan University, Wuhan 430072, China

S. Li $(\bowtie)$

Faculty of Mathematics and Statistics, Hubei Key Laboratory of Mathematical Science, Central China

Normal University, Wuhan 430079, China

e-mail: 1scmath@mail.ccnu.edu.cn 